\title{
The 26 year-long X-ray light curve and the X-ray spectrum of the BL Lacertae object 1E 1207.9+3945 in its brightest state
}

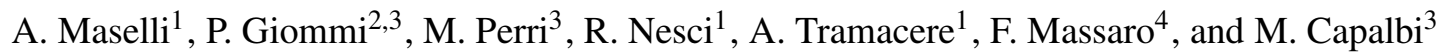 \\ 1 Dipartimento di Fisica, Università La Sapienza, Piazzale A. Moro 2, 00185 Roma, Italy \\ e-mail: alessandro.maselli@uniroma1.it \\ 2 Agenzia Spaziale Italiana, Unità Osservazione dell’Universo, Viale Liegi 26, 00198 Roma, Italy \\ 3 ASI Science Data Center, ESRIN, via G. Galilei, 00044 Frascati, Italy \\ 4 Dipartimento di Fisica, Università Tor Vergata, via della Ricerca Scientifica 1, 00133 Roma, Italy
}

Received 2 August 2007 / Accepted 24 September 2007

\section{ABSTRACT}

\begin{abstract}
Aims. We studied the temporal and spectral evolution of the synchrotron emission from the high energy peaked BL Lac object 1E $1207.9+3945$

Methods. Two recent observations have been performed by the XMM-Newton and Swift satellites; we carried out X-ray spectral analysis for both of them, and photometry in optical-ultraviolet filters for the Swift one. Combining the results thus obtained with archival data we built the long-term X-ray light curve, spanning a time interval of 26 years, and the Spectral Energy Distribution (SED) of this source.

Results. The light curve shows a large flux increasing, by about a factor of six, in a time interval of a few years. After reaching its maximum in coincidence with the XMM-Newton pointing in December 2000 the flux decreased in later years, as revealed by Swift. The very good statistics available in the $0.5-10 \mathrm{keV}$ XMM-Newton X-ray spectrum reveals a highly significant deviation from a single power law. A log-parabolic model with a best fit curvature parameter of 0.25 and a peak energy at $\sim 1 \mathrm{keV}$ describes well the spectral shape of the synchrotron emission. The simultaneous fit of Swift UVOT and XRT data provides a milder curvature $(b \sim 0.1)$ and a peak at higher energies $(\sim 15 \mathrm{keV})$, suggesting a different state of source activity. In both cases UVOT data support the scenario of a single synchrotron emission component extending from the optical/UV to the X-ray band.

Conclusions. New X-ray observations are important to monitor the temporal and spectral evolution of the source; new generation $\gamma$-ray telescopes like AGILE and GLAST may for the first time detect its inverse Compton emission.
\end{abstract}

Key words. radiation mechanisms: non-thermal - galaxies: active - X-rays: individuals: 1E 1207.9+3945 - X-rays: galaxies

\section{Introduction}

BL Lac objects are thought to be radio-loud Active Galactic Nuclei (AGNs) observed in a direction very close to the axis of a relativistic jet outflowing from the inner nuclear region (Urry $\&$ Padovani 1995). This interpretation could explain most of the characteristics of these sources like compact and flat-spectrum radio emission, superluminal motion revealed by VLBI imaging, high and variable radio and optical polarization, non-thermal continuum emission extending from radio to $\gamma$-ray frequencies, an almost featureless optical spectrum and the fast variability at all frequencies.

BL Lac objects are generally characterized by a double bump structure in the broad band Spectral Energy Distribution (SED). The low frequency bump is attributed to synchrotron radiation emitted by relativistic electrons in the jet; inverse Compton scattering by the same electron population on the synchrotron radiation is thought to be the origin of the high frequency bump. The peak of the first bump may vary over a rather wide range of frequencies: from the IR/optical band for the low energy peaked BL Lacs (LBLs) to the UV/X-ray band for the high energy peaked BL Lacs (HBLs) (Giommi \& Padovani 1994; Padovani \& Giommi 1995).

1E 1207.9+3945, also named BZB J1210+3929 in the recent Multifrequency Catalogue of Blazars (E. Massaro et al. 2007, www.asdc.asi.it/bzcat) is one of the X-ray selected BL Lacertae objects of the Einstein Medium Sensitivity Survey
(Gioia et al. 1990). It was discovered as a serendipitous source located about five arc-minutes north of one of the most intensively studied AGNs, the bright Seyfert galaxy NGC 4151. For this reason $1 \mathrm{E} 1207.9+3945$ has been observed on many occasions by all the imaging X-ray instruments that have operated since the Einstein observatory. Despite its relatively high redshift $(z=0.615)$ HST was able to detect the bright $\left(M_{\mathrm{R}}=-24.4\right)$ host galaxy which is of elliptical type (Scarpa et al. 2000).

In this paper we report the long-term X-ray light curve which spans 26 years. We also report and compare the spectral analysis of the most recent observations carried out by the XMM-Newton (Jansen et al. 2001) and Swift (Gehrels et al. 2004) satellites; we discuss the possibility of modeling the synchrotron emission of this source with a single log-parabolic model. The peak of the synchrotron component lies in the X-ray band, and for this reason the source can safely be classified as an HBL. We finally report the Spectral Energy Distribution (SED) of 1E 1207.9+3945 compiled from non-simultaneous multi-frequency archival data.

\section{The long-term X-ray light curve}

Because of its spatial proximity to the well known Seyfert Galaxy NGC 4151, 1E 1207.9+3945 has been observed by a large number of X-ray instruments on board several astronomical spacecraft. We collected all available historical X-ray data 
Table 1. Basic information on XMM-Newton and Swift observations. To distinguish the two different XMM-Newton exposures, both performed on 22 December 2000, we indicate with I the longer exposure, and with II the other one.

\begin{tabular}{ccccccc}
\hline \hline Satellite & Instrument & $\begin{array}{c}\text { Sequence } \\
\text { number }\end{array}$ & $\begin{array}{c}\text { Readout } \\
\text { mode }\end{array}$ & Date & $\begin{array}{c}\text { Exposure } \\
(\mathrm{s})\end{array}$ & Net counts \\
\hline XMM-Newton & EPIC-MOS1 & $\begin{array}{c}0112830201 \\
(\mathrm{I})\end{array}$ & $\begin{array}{c}\text { Full Window } \\
\text { Medium Filter }\end{array}$ & 22 Dec. 2000 & 59361 & 34236 \\
XMM-Newton & EPIC-MOS1 & $\begin{array}{c}0112830501 \\
(\text { II) }\end{array}$ & $\begin{array}{c}\text { Full Window } \\
\text { Medium Filter }\end{array}$ & 22 Dec. 2000 & 22092 & 12418 \\
Swift & XRT & 00035463001 & Photon Counting & 26 Dec. 2005 & 10368 & 1218 \\
\hline
\end{tabular}

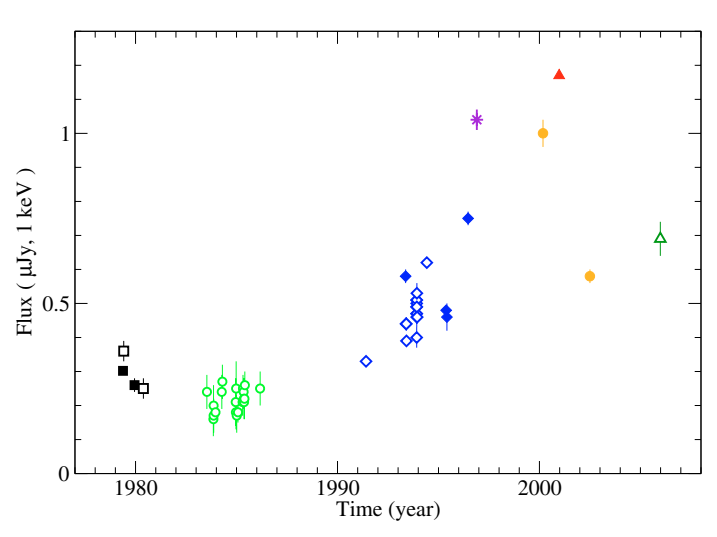

Fig. 1. The long-term X-ray light curve of 1E 1207.9+3945. Starting from the earliest observations we plotted data from Einstein IPC (filled squares) and HRI (empty squares), EXOSAT CMA (empty circles), ROSAT PSPC (empty diamonds) and HRI (filled diamonds), BeppoSAX MECS (star), Chandra (filled circles), XMM-Newton EPIC MOS1 (filled triangle) and Swift XRT (empty triangle) instruments.

from the literature and we also added more recent results from XMM-Newton, Chandra and Swift observations.

Most of the available data were accessed through the ASI Science Data Center (ASDC) on-line services (www.asdc.asi.it). Data relative to Einstein IPC were taken from the 2E catalog (Harris et al. 1994) while those relative to Einstein HRI come from the Einstein Observatory HRI source list (Giacconi et al. 1979). Data from ROSAT PSPC were derived from WGACAT2 catalog (White et al. 2000); data from ROSAT HRI were obtained from the BMW catalog (Panzera et al. 2003). We completed this information with those from EXOSAT CMA reported in Giommi et al. (1990). All these measurements, expressed in cts/s, were converted to monochromatic fluxes at $1 \mathrm{keV}$; each value has also been corrected for Galactic absorption adopting $N_{\mathrm{H}}=2.0 \times 10^{20} \mathrm{~cm}^{-2}$, a value estimated from $21 \mathrm{~cm}$ measurements along the line of sight (Dickey \& Lockman 1990).

The MECS instrument on board the BeppoSAX satellite observed 1E 1207.9+3945 in July and December 1996. As Cusumano et al. (2001) found no relevant change in the source flux over this time interval, they performed a spectral analysis summing the four available observations in a single spectral file; we report the flux at $1 \mathrm{keV}$ derived from the results of their spectral analysis. As regards the most recent observations (from XMM-Newton, Chandra and the latest by Swift) we performed data reduction and derived the flux at $1 \mathrm{keV}$ directly from the spectral fit. The light curve thus obtained spans a very long time interval, more than 25 years, and is plotted in Fig. 1.

The light curve is characterized by the following behaviour: the flux remained low $(0.2-0.3 \mu \mathrm{Jy})$ for $\mathrm{a} \simeq 10$ year-long time interval during the ' $80 \mathrm{~s}$, and then it increased during the ' $90 \mathrm{~s}$ to a high state, maintaining a level around $1 \mu \mathrm{Jy}$ for about four years. The highest flux was detected by XMM-Newton by the end of 2000, and it is about a factor of six higher than the minimum. After reaching its peak the source luminosity decreased, with the flux at the epoch of the most recent Swift pointing (December 2005) at a level comparable to the one detected by ROSAT ten years earlier, about three times higher than the minimum. Superposed on this trend, flux variations of smaller amplitude $(\sim 0.1 \mu \mathrm{Jy})$ and much shorter time scales (even 1-2 days) have been revealed thanks to the very close sequence of observations (one day frequency) in December 1993 by the ROSAT satellite.

\section{XMM-Newton observations}

1E 1207.9+3945 was observed by XMM-Newton on 22 December 2000, by the EPIC-PN (Struder et al. 2001) and EPIC-MOS (Turner et al. 2001) CCD cameras, operating in "full window" frame with medium filters. We restricted our analysis to the EPIC-MOS data only because for other HBL sources the estimates of the spectral parameters were found consistent with those obtained from BeppoSAX and Swift observations (Tramacere et al. 2007; F. Massaro et al. 2007). We checked both MOS1 and MOS2 data, and after verifying the consistency between results we report only those relative to MOS1. The pointing was split in two intervals, one significantly longer than the other; details are given in Table 1 . Both these observations were reduced adopting standard criteria and following the "User's Guide to the XMM-Newton Science Analysis System (SAS)" (Issue 3.1) (Loiseau et al. 2004) and "The XMM-Newton ABC Guide" (vers. 2.01) (Snowden et al. 2004). Particular care was dedicated to the solar flare subtraction, as described in Tramacere et al. (2007a).

Photons for spectral analysis were extracted from a circular region with a radius of $40^{\prime \prime}$. The background spectrum was extracted from a circular region of size comparable to the source region, in a place where visible sources were not present. The Photon Redistribution Matrix and the Ancillary Region File were created for each observation, by using the RMFGEN and ARFGEN tasks of SAS.

To ensure the validity of Gaussian statistics we grouped data so that each new bin included at least 40 counts. Spectral analysis was carried out using XSPEC 11.3.2 (Arnaud et al. 1996) in the $0.5-10 \mathrm{keV}$ restricted energy range to avoid possible residual calibration uncertainties. We separately analyzed the two observations and we found best fit parameters consistent within the errors, indicating that the source spectrum did not change significantly on such a short time scale; we thus applied a simultaneous fit of the two spectra.

First, we fitted the two data sets using a single power law model:

$F(E)=K E^{-a} \quad \mathrm{ph} \mathrm{cm}^{-2} \mathrm{~s}^{-1} \mathrm{keV}^{-1}$ 
Table 2. Best fit spectral parameters for XMM-Newton and Swift observations derived from XSPEC analysis. Statistical errors are taken at $1 \sigma$ confidence level. For each satellite we reported the results obtained adopting a power law model with fixed and free $N_{\mathrm{H}}$, and the log-parabolic model. We also added results derived fixing the curvature parameter to the value $(b=0.18)$ obtained from fitting non simultaneous Swift UVOT and XMM-Newton fluxes (see Sect. 6).

\begin{tabular}{cccccccc}
\hline \hline Satellite & $\begin{array}{c}N_{\mathrm{H}} \\
\left(10^{20} \mathrm{~cm}^{-2}\right)\end{array}$ & $\begin{array}{c}K \\
\left(10^{-3} \mathrm{ph} \mathrm{cm}^{-2} \mathrm{~s}^{-1} \mathrm{keV}^{-1}\right)\end{array}$ & $a$ & $b$ & $\begin{array}{c}E_{p} \\
(\mathrm{keV})\end{array}$ & $\begin{array}{c}F_{2-10} \\
\left(10^{-12} \mathrm{erg} \mathrm{cm}^{-2} \mathrm{~s}^{-1}\right)\end{array}$ & $\chi_{r}^{2} /$ d.o.f. \\
\hline XMM & 2.0 (fixed) & $1.74 \pm 0.01$ & $2.10 \pm 0.01$ & & & 3.8 & $1.23 / 376$ \\
XMM & $5.3 \pm 0.4$ & $1.92 \pm 0.03$ & $2.21 \pm 0.02$ & & & 3.6 & $1.07 / 375$ \\
XMM & 2.0 (fixed) & $1.76 \pm 0.01$ & $2.00 \pm 0.01$ & $0.25 \pm 0.03$ & 1.0 & 3.5 & $1.01 / 375$ \\
\hline XMM & 2.0 (fixed) & $1.76 \pm 0.01$ & $2.03 \pm 0.01$ & 0.18 (fixed) & 0.8 & 3.5 & $1.02 / 376$ \\
\hline Swift & 2.0 (fixed) & $0.96 \pm 0.03$ & $1.91 \pm 0.04$ & & & 2.8 & $1.34 / 52$ \\
Swift & $7.9 \pm 1.7$ & $1.22 \pm 0.08$ & $2.16 \pm 0.09$ & & & 2.5 & $1.09 / 51$ \\
Swift & 2.0 (fixed) & $1.04 \pm 0.04$ & $1.77 \pm 0.06$ & $0.43 \pm 0.13$ & 1.9 & 2.4 & $1.12 / 51$ \\
\hline
\end{tabular}

with the hydrogen equivalent column density fixed at the Galactic value: we found $\chi_{r}^{2} /$ d.o.f. $=1.23 / 376$ and residuals showing evidence of an intrinsic curvature. Repeating the fit with the same model and leaving $N_{\mathrm{H}}$ free to vary, we found a fit statistical improvement $\left(\chi_{r}^{2} /\right.$ d.o.f. $\left.=1.07 / 375\right)$ but the absorbing column density value was almost a factor of three $\left(N_{\mathrm{H}}=5.3 \times\right.$ $10^{20} \mathrm{~cm}^{-2}$ ) higher than the Galactic one. All parameter values relative to the spectral analysis are reported in Table 2 . We also used a broken power-law model with Galactic $N_{\mathrm{H}}$ and found a good fit $\left(\chi_{r}^{2} /\right.$ d.o.f. $\left.=0.98 / 374\right)$ with spectral photon indices $a_{1}=2.01 \pm 0.01$ and $a_{2}=2.34 \pm 0.04$ before and after the energy break $E_{\mathrm{b}}=2.2_{-0.3}^{+0.1} \mathrm{keV}$, respectively.

An alternative model that provides a good fit is given by the log-parabolic model (Massaro et al. 2004a):

$$
F(E)=K\left(E / E_{0}\right)^{-\left(a+b \log \left(E / E_{0}\right)\right)} \quad \mathrm{ph} \mathrm{cm}^{-2} \mathrm{~s}^{-1} \mathrm{keV}^{-1}
$$

where $a$ is the spectral index (given by the log-derivative) at $E_{0}=$ $1 \mathrm{keV}$ and $b$ measures the spectral curvature. This spectral model is able to represent an intrinsic curved spectra with the addition of just one parameter to the single power law; it has been successfully applied to modelling the SED of other HBL sources like e.g. Mrk 421 (Massaro et al. 2004a), Mrk 501 (Massaro et al. 2004b), PKS 0548-322 (Perri et al. 2007) and also a sample of HBLs detected at Tev energies (Tramacere et al. 2007b). Moreover, it allows us to estimate in a very simple way other interesting quantities, like the energy of the SED peak given by the following expression:

$E_{p}=E_{0} 10^{(2-a) / 2 b} \mathrm{keV}$.

The log-parabolic model provides for 1E $1207.9+3945$ a very good $\chi_{r}^{2}$ value $\left(\chi_{r}^{2} /\right.$ d.o.f. $\left.=1.01 / 375\right)$ and a curvature parameter $(b=0.25 \pm 0.03)$ consistent with typical values obtained in the spectral analysis of other HBL sources (all parameter values are reported in Table 2). Figure 2 reports the spectrum and the residuals of the analysis in the whole range $(0.5-10 \mathrm{keV})$.

To estimate the significance of the improvement of the power law model with free $N_{\mathrm{H}}$ and the log-parabolic model with respect to the power law model with Galactic $N_{\mathrm{H}}$, we applied an F-test. In both cases we obtained very low values $\left(5.0 \times 10^{-13}\right.$ and $8.6 \times 10^{-18}$, respectively) so that the two alternative models are significantly better than the power law with a Galactic $N_{\mathrm{H}}$ value. The log-parabolic model has the additional advantage that it does not require any intrinsic absorption, originating in the innermost nuclear environment or in the host galaxy, to justify the spectral curvature.

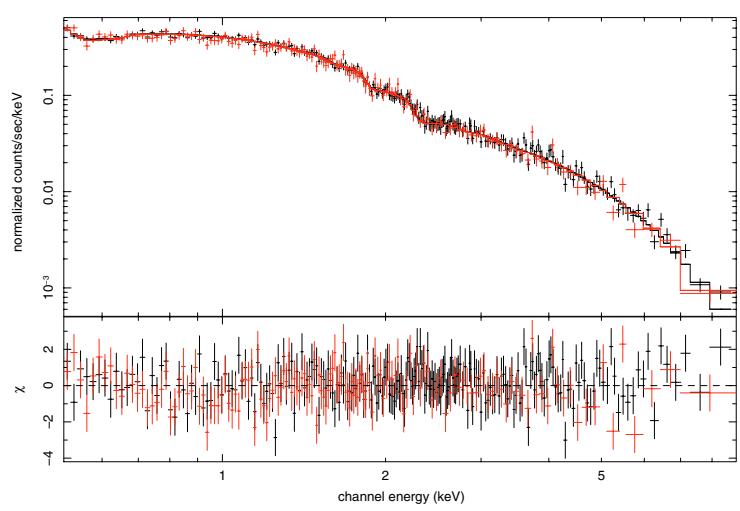

Fig. 2. The simultaneous fit of the XMM-Newton observations I and II adopting a log-parabolic law in the $0.5-10 \mathrm{keV}$ energy range; the $N_{\mathrm{H}}$ parameter is fixed to the known Galactic value.

\section{Swift observation}

1E 1207.9+3945 was observed in the field of NGC 4151 on 26 December 2005 with both the UVOT (UltraViolet-Optical Telescope, Roming et al. 2005) and XRT (X-Ray Telescope, Burrows et al. 2005) instruments.

The XRT observation was carried out using the most sensitive Photon Counting readout mode (see Hill et al. 2004, for a description of readout modes). Data were reduced with the XRTDAS software package (version 2.1.2) developed at the ASDC (Capalbi et al. 2005) and distributed within the HEASOFT 6.3 package by the NASA High Energy Astrophysics Archive Research Center (HEASARC).

We used the XIMAGE task to detect the source count rate and the centroid, and to choose a nearby source-free region in order to extract the background spectrum. The source count rate was $\sim 0.2 \mathrm{cts} / \mathrm{s}$, a value not high enough $(\geq 0.5 \mathrm{cts} / \mathrm{s})$ to determine photon pile-up occurrence. Spectral data were then extracted in a circular region within a 20 pixel radius $\left(1\right.$ pixel $\left.=2.36^{\prime \prime}\right)$ from the centroid; the background spectrum was estimated in a circular region of 50 pixels radius.

We then used the XRTPRODUCTS task to obtain all the files necessary for the spectral analysis; the exposure map was taken into account to correct for vignetting, CCD hot and damaged pixels. In the spectral file, instrumental channels were combined to include at least 20 counts in each new energy bin. The spectral analysis was carried out in the $0.3-10 \mathrm{keV}$ energy range using XSPEC 11.3.2.

Following the same criteria described in Sect. 3 we first fit the data using a single power law model, both fixing the hydrogen equivalent column density to the Galactic value and 


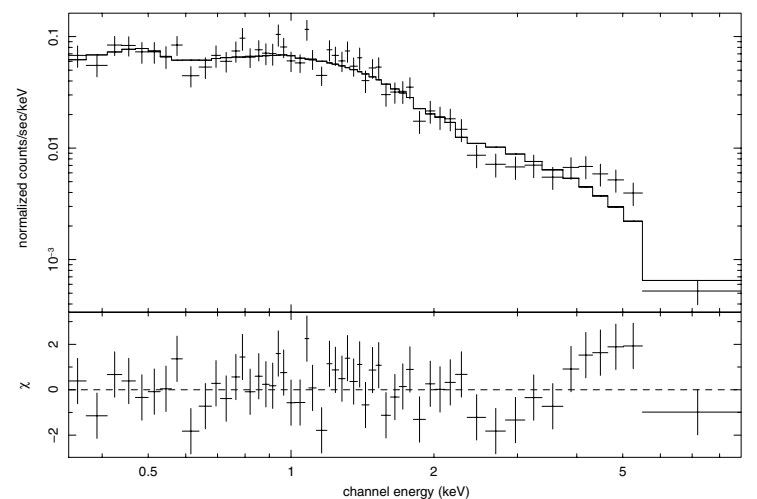

Fig. 3. The spectral analysis of the Swift XRT observation in the $0.3-10 \mathrm{keV}$ energy range adopting a log-parabolic model and fixing the $N_{\mathrm{H}}$ parameter to the known Galactic value.

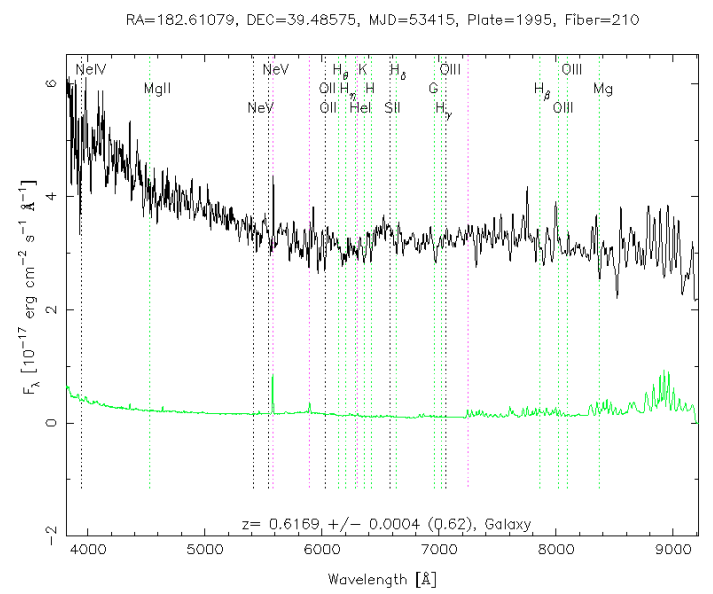

Fig. 4. The optical spectrum from SDSS, with the evident change in the spectral slope at $\lambda \simeq 6000 \AA$.

leaving it free to vary (see Table 2 ). In the second case we obtained, as expected, a better $\chi_{r}^{2}$ value $\left(\chi_{r}^{2} /\right.$ d.o.f. $=1.09 / 51 \mathrm{vs}$. $1.34 / 52)$ but an $N_{\mathrm{H}}$ value almost a factor of four $\left(N_{\mathrm{H}}=7.9 \times\right.$ $10^{20} \mathrm{~cm}^{-2}$ ) higher than the Galactic one. Again we fit the logparabolic model with Galactic $N_{\mathrm{H}}$ to data, obtaining a comparable improvement $\left(\chi_{r}^{2} /\right.$ d.o.f. $\left.=1.12 / 51\right)$ with no extra absorption needed (see Table 2 ). The F-test gives a probability of $2.62 \times$ $10^{-3}(\sim 3 \sigma)$ that this improvement is due to chance.

The spectrum and the residuals are plotted in Fig. 3. The distinctive feature of this plot is a dip around $3 \mathrm{keV}$ followed by an apparent rise at higher energies. New Swift observations with an exposure time longer than $10 \mathrm{ks}$ would help reduce the fluctuations characteristic of this spectrum.

\subsection{UVOT data reduction}

The UVOT instrument obtained a series of images in each of the lenticular filters $V, B, U, W 1, M 2, W 2$. The data analysis was performed using dedicated tasks included in the HEASOFT 6.3 package. Each series was summed with the UVOTIMSUM task to obtain a single frame in each filter; photometry was then performed using the UVOTSOURCE task.

Following the UVOT Team prescriptions (Immler et al. 2006) we chose an aperture radius of $2^{\prime \prime}$ for photometry, independently of the image filter; then we applied an aperture correction to transfer the obtained magnitude to the standard photometry aperture that was used to obtain the photometric zero point,
Table 3. 1E 1207.9+3945 de-reddened magnitudes in the UVOT six filters, and corresponding specific fluxes.

\begin{tabular}{ccc}
\hline \hline Filter & $\begin{array}{c}\text { Magnitude } \\
(\mathrm{mag})\end{array}$ & $\begin{array}{c}\text { Specific flux } \\
(\mu \mathrm{Jy})\end{array}$ \\
\hline$V$ & $19.76 \pm 0.19$ & $39.2 \pm 6.9$ \\
$B$ & $20.30 \pm 0.24$ & $28.9 \pm 6.4$ \\
$U$ & $18.90 \pm 0.09$ & $36.4 \pm 3.0$ \\
$W 1$ & $18.85 \pm 0.08$ & $27.3 \pm 2.0$ \\
$M 2$ & $18.87 \pm 0.09$ & $26.0 \pm 2.1$ \\
$W 2$ & $18.98 \pm 0.08$ & $24.6 \pm 1.8$ \\
\hline
\end{tabular}

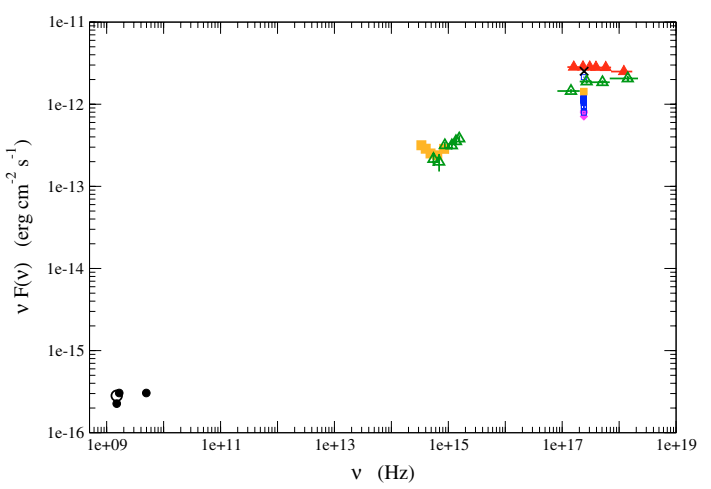

Fig. 5. The Spectral Energy Distribution of 1E 1207.9+3945. Filled black circles represent simultaneous radio data taken at the VLA (Stocke et al. 1985) while the empty circle corresponds to the FIRST pointing; the nearly coincident value in NVSS has not been plotted for simplicity. Filled orange squares representing SDSS data overlap Swift UVOT empty triangles (dark green) in the optical region of the spectrum. At $1 \mathrm{keV}$ energy, Einstein IPC are plotted in magenta (filled diamonds) and ROSAT PSPC in blue (empty squares); the orange filled square corresponds to the detection in the ROSAT All Sky Bright Source Catalog (1RXS) (Voges et al. 1999) while the black cross corresponds to BeppoSAX. The same symbol as UVOT has been used to plot Swift XRT data; red filled triangles refer to the XMM-Newton observation I.

which is $6^{\prime \prime}$ for $V, B, U$ filters and $12^{\prime \prime}$ for the $W 1, M 2, W 2$ filters. To achieve this purpose we selected a sample of stellar sources with a high signal-to-noise ratio and we calculated the difference between the magnitudes measured with the two different radii. After deriving a mean value for the aperture correction in each filter we obtained the corrected magnitudes for 1E 1207.9+3945.

The background region was chosen in the form of an annulus around the extraction region, with inner and outer radii taken to avoid the inclusion of spurious sources, particularly numerous in $U V$ filter frames; typical values are respectively $18^{\prime \prime}$ and $23^{\prime \prime}$. Then the obtained magnitudes were de-reddened using the value of $E(B-V)=0.03 \mathrm{mag}$ (Schlegel et al. 1998) and adopting the extinction curve given in Seaton (1979) with $R_{V}=3.2$, and finally converted to specific fluxes; results are reported in Table 3 .

\section{Optical spectrum and host galaxy contribution}

1E $1207.9+3945$ is present in the POSS-I and POSS-II plates at a very faint level, very close to the plate sensitivity limits ( $B$ and $R \simeq 20$ ), and no appreciable variation is apparent between the two epochs. The source is also present in the Sloan Digital Sky Survey (SDSS) and has been observed on 17 February 2004; also in this case no significant variation can be found comparing magnitudes in $\mathrm{g}$ and $\mathrm{r}$ filters to magnitudes in the POSS $B$ and $R$ filters. The SDSS optical spectrum (http://cas.sdss.org) is reported in Fig. 4: it does not show evidence of prominent lines 


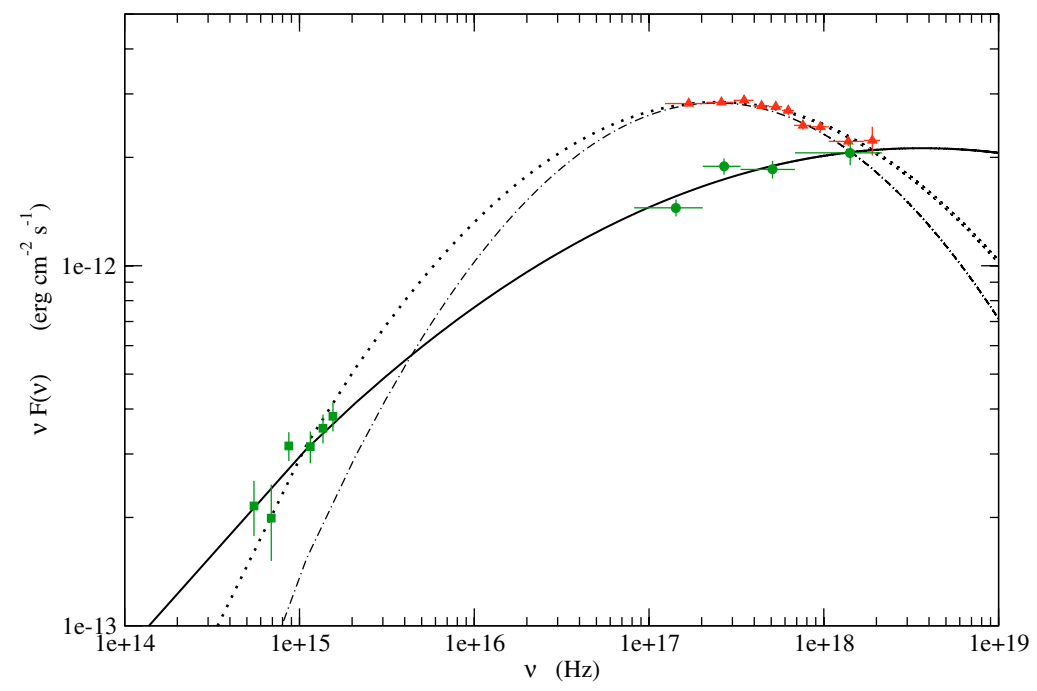

Fig. 6. An optical to X-ray SED of 1E 1207.9+3945 including Swift UVOT (squares), Swift XRT (circles) and XMM-Newton MOS1 (triangles) data sets. Note that the X-ray data have been further rebinned in XSPEC than by the criteria followed in the spectral analysis and described in Sects. 3 and 4. A log-parabolic model has been fitted to different data sets: solid line $(b \sim 0.1)$ represents the best fit to Swift UVOT and XRT simultaneous fluxes; dot-dashed line $(b \sim 0.25)$ represents the best fit to the XMM-Newton data points, extended to a wider frequency interval; dotted line $(b \sim 0.18)$ instead represents the best fit to non simultaneous UVOT and XMM-Newton (observation I) data sets.

and presents a slope change around $\lambda \simeq 6000 \AA$. This variation can be interpreted as the sign of the combined BL Lac and host galaxy emission: while the first one dominates at higher frequencies, its contribution is overwhelmed by the host galaxy at higher wavelengths. The host galaxy contribution clearly emerges also from the inspection of the Spectral Energy Distribution (SED) in the frequency interval $10^{14}<v<10^{15} \mathrm{~Hz}$ (see Fig. 5). To verify the agreement between the information in the optical spectrum and in the SED we graphically estimated the slope variation in the two different plots. We divided the SDSS spectrum at $6000 \AA$ in two intervals and graphically estimated the slope change on both sides. Analogously, we took SDSS points in the SED and split them in two sets, corresponding to the IR-optical $(z, i, r)$ and optical-UV $(g, u)$ filters; we traced a line interpolating the two different sets and estimated their slope and the relative variation. The same value of $\Delta \alpha \simeq 1.2$ that we found in both cases supports the interpretation that the host galaxy is mostly responsible for the infrared emission visible in the SED.

\section{Discussion}

Adding the results of our analysis to multi-frequency archival data we built the radio to X-ray SED of 1E 1207.9+3945 (Fig. 5) which follows the characteristic trend of extreme HBL sources (Padovani \& Giommi 1995) with the synchrotron emission covering the entire frequency interval and peaking well into the $\mathrm{X}$-ray band. The inverse Compton component is not detected and therefore is expected at higher energies.

The Swift satellite is sensitive both in the X-ray and in the optical-ultraviolet band. This provides the opportunity to test whether the Spectral Energy Distribution of a source like 1E $1207.9+3945$ can be explained by a single synchrotron component, or whether multiple emission components are present. For this we first fitted a log-parabolic model to simultaneous Swift XRT and UVOT fluxes (solid line in Fig. 6) and obtained a rather low value for the curvature parameter $(b \sim 0.1)$, in contrast with the one obtained by fitting XRT data only, as reported in Table 2; the energy peak lies at about $15 \mathrm{keV}$ or more. Comparing these results with those from XMM-Newton, Swift evidently caught 1 E $1207.9+3945$ in a different state of activity, characterized by a milder curvature and a peak of the synchrotron component shifted to higher energies. Firm conclusions cannot be drawn due to the relatively poor statistics and to the fact that the $E_{p}$ value obtained likely lies at energies higher than $10 \mathrm{keV}$, outside the range of Swift XRT.

Although aware of the limits of using non simultaneous observations for a variable source, we verified whether Swift UVOT and XMM-Newton MOS1 data are compatible with a logparabolic model. We took points representing the XSPEC model of observation I and fit them over a wider frequency interval (dot-dashed line in Fig. 6): UVOT points are systematically above the extrapolation of the log-parabolic model. We took care to compare this result with the one obtained by directly fitting X-ray rebinned data and obtained an almost coincident result. Then we fitted both data sets with a log-parabola (dotted line in Fig. 6) and estimated the curvature parameter: we obtained $b=0.18 \pm 0.01$, a value at about $2 \sigma$ of the one derived fitting only XMM-Newton data (see Table 2). At this point we tested the log-parabolic model with $b$ fixed at 0.18 and found $\chi_{r}^{2} /$ d.o.f. $=1.02 / 376$, a value practically coincident with the one obtained leaving $b$ free to vary, with only a small difference in the $a$ parameter. This result encouraged us to conclude that a single synchrotron component is in good agreement with the emission observed.

1E $1207.9+3945$ is worth monitoring in coming years. New observations in the X-ray band would add essential information to the light curve behaviour: if any kind of regularity in flux increasing and fading should emerge, it would be possible to place physical constraints to establish the nature of the mechanism responsible for these variations. Moreover, observations with instruments that extend to higher energies than XRT, like those on board Suzaku, would extend the known Spectral Energy Distribution and would allow us to obtain a better parametrization of the spectral curvature. Finally, 1E 1207.9+3945 may be an interesting target for AGILE and the forthcoming GLAST $\gamma$-ray mission which could detect for the first time the inverse Compton component of this HBL source. 
Acknowledgements. We thank Enrico Massaro for useful discussions and helpful comments, Gino Tosti and Simonetta Puccetti for hints on Swift UVOT and Chandra data analysis respectively. The authors acknowledge the financial support by the Italian Space Agency (ASI) for ASDC and the Physics Department of Università di Roma "La Sapienza".

\section{References}

Arnaud, K. A. 1996, Astronomical Data Analysis Software and Systems V, ed. G. Jacoby, \& J. Barnes, ASP Conf. Ser., 101, 17

Burrows, D. N., Hill, J. E., Nousek, J. A., et al. 2005, Space Sci. Rev., 120, 165 Capalbi, M., Perri, M., Saija, B., Tamburelli, F., \& Angelini, L. 2005, The Swift XRT Data Reduction Guide, http://heasarc.gsfc.nasa.gov/ docs/swift/analysis/xrt_swguide_v1_2.pdf

Cusumano, G., Mineo, T., \& Giommi, P. 2001, Astro. Lett. Commun., 40, 109

Dickey, J., \& Lockman, F. 1990, A\&A, 28, 215

Gehrels, N., Chincarini, G., Giommi, P., et al. 2004, ApJ, 611, 1005

Giacconi, R., Branduardi, G., Briel, U., et al. 1979, The Einstein Observatory, HEAO-2

Gioia, I. M., Maccacaro, T., Schild, R. E., et al. 1990, ApJS, 72, 567

Giommi, P., \& Padovani, P. 1994, MNRAS, 268, L51

Giommi, P., Barr, P., Garilli, B., et al. 1990, ApJ, 356, 432

Harris, D. E., et al. 1994, SAO HEAD CD-ROM Series I, Einstein, Nos. 18-36

Hill, J. E., Burrows, D. N., Nousek, J. A., et al. 2004, Proc. SPIE, 5165, 217

Immler, S., et al. 2006, The Swift UVOT Software guide, version 2.0
Jansen, F., Lumb, D., Altieri, B., et al. 2001, A\&A, 365, L1

Loiseau, N., et al. 2004, User's guide to the XMM-Newton Science Analysis System

Massaro, E., Perri, M., Giommi, P., \& Nesci, R. 2004a, A\&A, 413, 489

Massaro, E., Perri, M., Giommi, P., Nesci, R., \& Verrecchia, F. 2004b, A\&A, 422, 103

Massaro, E., Sclavi, S., Giommi, P., et al. 2007, www .asdc.asi.it/bzcat

Massaro, F., Tramacere, A., Cavaliere, A., Perri, M., \& Giommi, P. 2007, A\&A, submitted

Padovani, P., \& Giommi, P. 1995, ApJ, 444, 567

Panzera, M. R., Campana, S., \& Covino, S. 2003, A\&A, 399, 351

Perri, M., Maselli, A., Giommi, P., et al. 2007, A\&A, 462, 889

Roming, P. W. A., Kennedy, T. E., Mason, K. O., et al. 2005, Space Sci. Rev., 120,95

Scarpa, R., Urry, C. M., Falomo, R., et al. 2000, ApJ, 532, 740

Schlegel, D. J., Finkbeiner, D. P., \& Davis, M. 1998, ApJ, 500, 525

Seaton, M. J. 1979, MNRAS, 187, 73

Stocke, J. T., Liebert, J., \& Schmidt, G. 1985, ApJ, 298, 619

Snowden, S., et al. 2004, The XMM-Newton ABC Guide, version 2.01

Struder, L., Briel, U., Dennerl, K., et al. 2001, A\&A, 365, L18

Tramacere, A., Massaro, F., \& Cavaliere, A. 2007a, A\&A, 466, 521

Tramacere, A., Giommi, P., Massaro, E., et al. 2007b, A\&A, 467, 501

Turner, M. L. J., Abbey, A., Arnaud, M., et al. 2001, A\&A, 365, L27

Urry, C. M., \& Padovani, P. 1995, PASP, 197, 803

Voges, W., Aschenbach, B., Boller, T., et al. 1999, A\&A, 349, 389

White, N. E., Giommi, P., Angelini, L., WGACAT 2000,

http://wgacat.gsfc.nasa.gov/wgacat/wgacat.html 